\title{
Utilización de stent autoexpandible en canino con colapso traqueal
}

\author{
López, M.L. ${ }^{1,2}$; Saravia, E.D.; Lockett, M.B. ${ }^{1}$ \\ ${ }^{1}$ Cátedra de Cirugía y Anestesiología, ${ }^{2}$ Hospital de Grandes Animales, \\ Facultad Ciencias Veterinarias, Univ.Nac.Nordeste, Corrientes, Argentina. \\ E-mail: mayritalopez2011@hotmail.com
}

\begin{abstract}
Resumen
López, M.L.; Saravia, E.D.; Lockett, M.B.: Utilización de stent autoexpandible en canino con colapso traqueal. Rev. Vet. 31: 1, 46-49, 2020. El colapso traqueal canino se presenta principalmente en razas pequeñas, entre los 5 y 9 años, pudiendo manifestar dificultad respiratoria, tos seca crónica, estertores, disnea, cianosis e incluso síncope. El tratamiento quirúrgico está indicado en pacientes que no responden a la medicación y presentan un grado de colapso avanzado (disminución de la luz traqueal del 50\% o más) y cuando tal disfunción ocurre en la porción intratorácica. La técnica utilizada en este caso fue la menos invasiva y de elección para pacientes de alto riesgo, consistente en la colocación de una endoprótesis autoexpandible en la luz traqueal. En el paciente Shitzu, de 14 años, $9 \mathrm{~kg}$ de peso, con colapso traqueal grado 5, se realizó videoendoscopía y radiografías digitalizadas para determinar la longitud y diámetro del stent apropiado. Se utilizó un stent metálico de platinol autoexpandible de $10 \mathrm{~mm}$ de diámetro por $100 \mathrm{~mm}$ de longitud. La recuperación anestésica fue rápida y tranquila, pudiendo retirarse el tubo endotraqueal al cabo de 5 minutos sin que se presentaran contratiempos. La mejoría respiratoria, luego de la finalización del procedimiento, fue inmediata y sin complicaciones.
\end{abstract}

Palabras clave: canino, colapso traqueal, stent, endoprótesis, mejoría respiratoria.

\begin{abstract}
López M.L.; Saravia, E.D.; Lockett, M.B.: Utilization of stent in canine tracheal collapse. Rev. Vet. 31: 1, 46-49, 2020. Tracheal collapse occurs mainly in small breed dogs, with ages ranging 5-9 years old. Symptoms may include breathing difficulty, chronic dry cough, rales, dyspnea, cyanosis and syncope. Surgical treatment is indicated in patients that do not respond to medication and have an advanced degree of tracheal collapse (reduction of tracheal lumen of $50 \%$ or more) and when collapse occurs in the intra-thoracic portion of the organ. Less invasive surgical techniques are preferred for high-risk patients, consisting in the placement of a self-expanding stent in the tracheal lumen. In a 14-year-old Shitzu dog, weighing $9 \mathrm{~kg}$, with grade 5 tracheal collapse, video-endoscopy and digitalized radiographs were performed to determine the length and diameter of the appropriate stent. A self-expanding platinol stent, $10 \mathrm{~mm}$ in diameter and $100 \mathrm{~mm}$ in length, was used. After surgery, recovery was quick, and the endotracheal tube could be removed after 5 minutes without difficulties. Respiratory improvement after surgery was immediate and without complications.
\end{abstract}

Key words: dog, tracheal collapse, stent, endoprosthesis, breathing improvement.

\section{INTRODUCCIÓN}

La tráquea es una estructura tubular semi-rígida, con cartílagos hialinos en forma de "C", unidos por ligamentos anulares elásticos y conectados dorsalmente por la membrana traqueal dorsal. La vascularización es segmentaria de pequeñas ramas procedentes de las arterias tiroideas craneales y caudales ${ }^{1,5}$.

El colapso traqueal es un tipo de obstrucción secundario a la flacidez de los cartílagos que pierden rigidez y son incapaces de mantener la forma de la tráquea du-

Recibido: junio 2019 / Aceptado: setiembre 2019 rante la respiración, colapsándose dorso-ventralmente. Esta alteración se presenta principalmente en razas pequeñas, con edades comprendidas entre los 5 y 9 años 5 . Los animales con colapso traqueal sufren síndrome de dificultad respiratoria, manifestado por tos crónica, discordante o seca, estertores similares al graznido del ganso, disnea respiratoria, cianosis e incluso síncope. El cuadro evoluciona progresivamente ${ }^{1,5}$.

Si la sintomatología del paciente induce a sospechar colapso traqueal, se deben llevar a cabo pruebas complementarias como radiografías en inspiración/espiración, fluoroscopía, tomografía computada y traqueobroncoscopía (siendo estas tres últimas una limitante 
en nuestro medio). El principal diagnóstico diferencial se debe realizar con estenosis traqueal ${ }^{5}$.

A pesar de que puede ser un problema mecánico o funcional que potencialmente puede ser mejorado e incluso corregido en ausencia de enfermedad parenquimatosa o sistémica subyacente, representa todo un desafío tanto para cirujanos como para neumólogos ${ }^{4}$.

El tratamiento quirúrgico está indicado en aquellos pacientes que no responden a la medicación y presentan un grado de colapso avanzado, con una reducción de la luz traqueal del $50 \%$ o más, especialmente cuando el colapso se presenta en la porción intra-torácica. Las técnicas se basan en brindar sostén a los cartílagos y músculo traqueal mediante prótesis extra-luminales o intra-luminales colocadas bajo técnicas quirúrgicas convencionales o de mínima invasión respectivamente, sin alterar el aclaramiento muco-ciliar ${ }^{2,5}$.

Para decidir el procedimiento quirúrgico a aplicar en cada caso, se debe valorar la localización del colapso, su grado, longitud y diámetro del mismo ${ }^{1,6}$. En relación al tratamiento convencional, la implantación de un stent metálico auto-expandible presenta las ventajas de ser un método rápido y mínimamente invasivo, que suprime la posibilidad de complicación quirúrgica por manipulación externa de la tráquea y produce una mejoría inmediata, reduciendo el tiempo de recuperación del paciente ${ }^{5}$. El objetivo del tratamiento es lograr una mejoría duradera. Numerosos estudios demuestran mejoría clínica posterior a la colocación de stent en el 90-97\% de los casos ${ }^{4}$.

En cuanto a la clasificación de los stents, debe tenerse en cuenta que existen variados modelos, de diferentes materiales. Las dos principales categorías son los fabricados de silicón y los de metal, pudiendo combinarse o bien emplearse otro tipo de material para fabricar modelos híbridos ${ }^{4}$. Existen dos diseños metálicos básicos: los auto-expandibles que poseen un diámetro predeterminado y una buena adaptación a los diferentes tamaños traqueales, y los expandibles con balón, que no poseen expansibilidad dinámica, y requieren ser expandidos activamente hasta el diámetro deseado ${ }^{4}$.
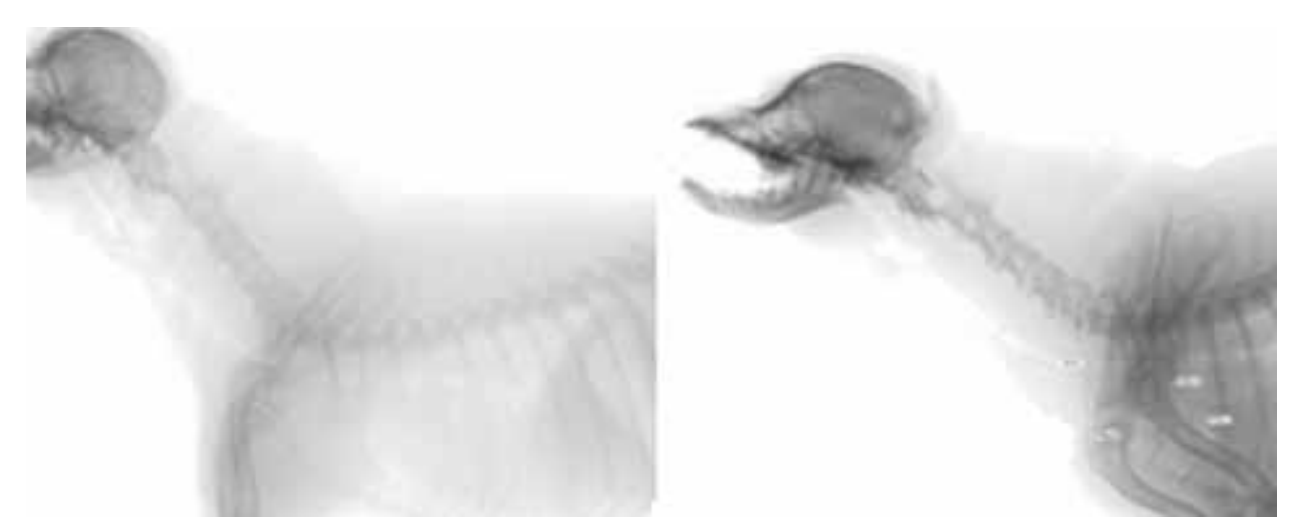

Figura 1. Izquierda: Rx cervicotorácica en inspiración/espiración, observándose disminución de la luz traqueal a nivel de las últimas vértebras cervicales y primeras torácicas. Derecha: mediciones para la determinación del stent adecuado.
El stent es fácil de liberar y colocar; posee suficiente fuerza radial para mantener la permeabilidad y evitar migraciones, alta elasticidad para evitar la fatiga del material, buena flexibilidad longitudinal, aceptable bio-compatibilidad para evitar la formación de tejido de granulación o infección y mantener el aclaramiento muco-ciliar. Al ser desplegado sufre un acortamiento del 20 al $40 \%$ de su longitud ${ }^{3,4}$.

El objetivo del presente trabajo fue comunicar un caso de colapso traqueal grave, en el que se realizó la técnica de colocación intra-luminal de una endo-prótesis de Wallstent.

\section{MATERIAL Y MÉTODOS}

El paciente canino fue una hembra castrada, de raza Shitzu, de 14 años de edad y $9 \mathrm{~kg}$ de peso, con colapso traqueal de grado 5 .

El método complementario realizado fue la radiografía digitalizada de la tráquea cervical y torácica en inspiración/espiración, a través de la cual se determinó la longitud y el diámetro del stent adecuado (Figura 1, izquierda y derecha).

El stent utilizado fue un Wallflex Biliary ${ }^{\mathrm{TM}}$ (Boston Scientific) de $10 \mathrm{~mm}$ de diámetro x $100 \mathrm{~mm}$ de longitud, con aleación metálica de platinol (núcleo de platino con revestimiento de nitinol), y una guía Hydra Jagwire ${ }^{\mathrm{TM}}$ de $0,89 \mathrm{~mm}$ de diámetro y $450 \mathrm{~cm}$ de longitud del mismo fabricante.

Para lograr la inducción anestésica, el protocolo utilizado (vía endovenosa) fue butorfanol $0,2 \mathrm{mg} / \mathrm{kg}$, ketamina $1 \mathrm{mg} / \mathrm{kg}$ y propofol $3 \mathrm{mg} / \mathrm{kg}$. Seguidamente se realizó la intubación endotraqueal con tubo Murphy número 4,5. En el mantenimiento se utilizó infusión continua de propofol y durante todo el procedimiento se suministró $\mathrm{O}_{2}$ a razón de $2 \mathrm{l} / \mathrm{min}$, manteniéndose un plano anestésico 2 del período 3 .

Se realizó el monitoreo básico con oximetría de pulso, cuyo sensor fue colocado a nivel de la lengua midiendo saturación de $\mathrm{O}_{2}$, pulso y curva pletismográfica. También se monitoreó la frecuencia cardíaca a intervalos regulares, mediante fonendoscopio. 


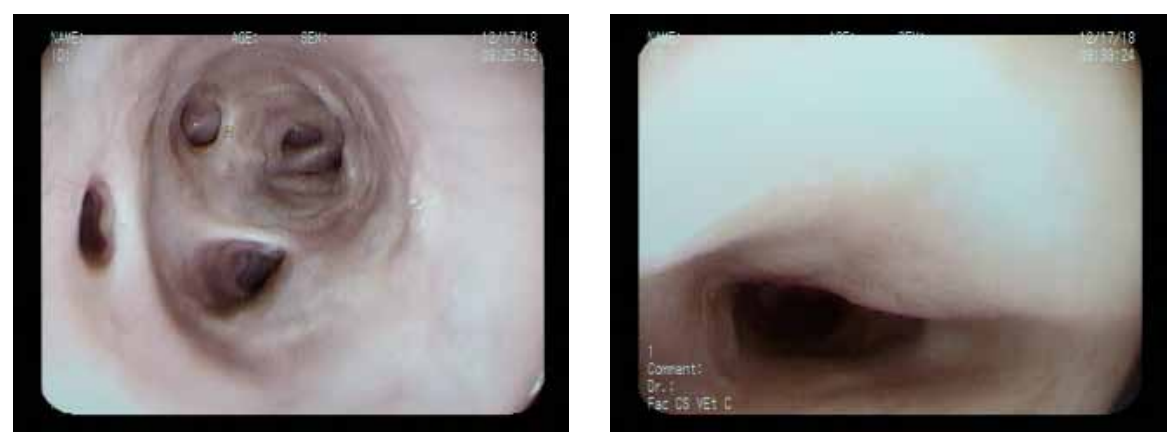

Figura 2. Izquierda: videoendoscopía a nivel de bronquios, sin colapso. Derecha: videoendoscopía hacia la zona craneal, observándose la luz traqueal colapsada. Ambas figuras del mismo paciente.

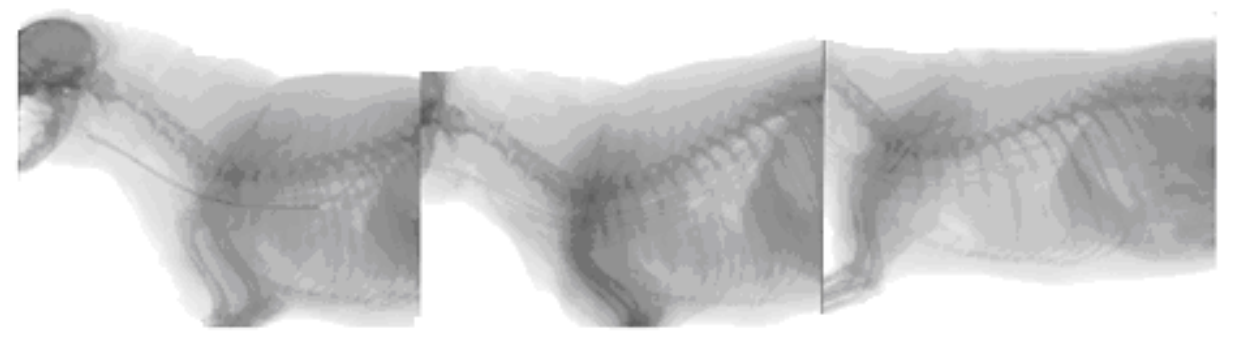

Figura 3. Izquierda: Rx donde se advierte la posición del stent sin desplegar dentro de la luz traqueal, observándose además la guía en el interior del esófago. Central: stent desplegado y guía de referencia. Derecha: se observa la correcta ubicación de la endoprótesis en la zona de colapso cervicotorácico.

Para introducir la sonda del video-endoscopio en la luz traqueal, se retiró transitoriamente el tubo de Murphy para determinar la severidad y extensión del colapso. Los hallazgos fueron documentados fotográficamente (Figura 2, izquierda y derecha). Debido a la mala saturación de $\mathrm{O}_{2}$ y dificultad respiratoria causado por el diámetro de la sonda de video-endoscopía en relación al diámetro de la luz traqueal, fue necesario en ese momento realizar una punción traqueal con un abbocath $\mathrm{N}^{\circ} 14$.

El paso siguiente fue la introducción de la guía ya mencionada (Hydra Jagwire ${ }^{T M}$ ) en la luz del esófago, como referencia para la obtención de placas radiográficas durante el procedimiento de colocación del stent en tráquea torácica y cervical.

A través del tubo endo-traqueal previamente reinsertado, se introdujo el dispositivo con la endo-prótesis en la luz traqueal, haciéndola avanzar hasta la zona más caudal del colapso, inmediatamente craneal a la carina traqueal; confirmándolo mediante radiografías seriadas. Luego se procedió al despliegue completo del stent una vez confirmada su correcta ubicación (Figura 3 , izquierda, central y derecha).

Luego del tratamiento quirúrgico se administró dexametasona $0,5 \mathrm{mg} / \mathrm{kg}$ y butorfanol $0,1 \mathrm{mg} / \mathrm{kg}$. para prevenir $y / o$ tratar la inflamación y tos respectivamente.

\section{RESULTADOS Y DISCUSIÓN}

Cabe señalar que las condiciones estaban dadas para la realización de este tipo de procedimiento, relativamente nuevo en nuestro medio y sobre todo en nuestra región, ya que no se tenía registro documentado o publicado de este tipo de intervención.

La recuperación anestésica fue rápida y tranquila, pudiendo retirarse el tubo endotraqueal al cabo de 5 minutos sin que se presentaran contratiempos. La notoria mejoría respiratoria del paciente luego de la finalización del procedimiento fue inmediata y sin complicaciones.

Para decidir la técnica a aplicar en cada caso, debe valorarse la localización del colapso, el grado, la longitud y el diámetro del mismo ${ }^{5}$. Esta opción quirúrgica deberá indicarse principalmente en pacientes con colapso intra-torácico extenso o cuando son malos candidatos para la cirugía convencional 1,2,4,5. El principal inconveniente del uso de stent metálico autoexpandible es la reactividad que provoca al interactuar con la mucosa traqueal ${ }^{1}$.

Dentro de las complicaciones frecuentes asociadas a la colocación de stent en las vías aéreas, se encuentran las trans-operatorias: mal posicionamiento, imposibilidad técnica para la colocación, perforación traqueo-bronquial, enfisema, neumotórax y sangrado. Las complicaciones agudas $(<72 \mathrm{~h})$ incluyen sangrado, disfonía, tos, migración, retención de secreciones y obstrucción. Las tardías ( $>72$ h) comprenden colonización o infección, shock séptico, formación de tejido de granulación, fatiga o fractura del metal, dolor $\mathrm{y} \mathrm{tos}^{4}$.

Agradecimientos. Al MV Manuel Martin Flores (Department of Clinical Sciences, College of Veterinary Medicine, Cornell, USA), por el asesoramiento médico. A la MV Mamonde Alexia, por colaborar en el desarrollo del procedimiento. Al Sr Guillermo Dohr- 
mann (empresa Endouro Medical) por el asesoramiento técnico.

\section{REFERENCIAS}

1. Brockman DJ, Holt DE. 2012. Manual de cirugía de cabeza, cuello y tórax en pequeños animales, Ed. Lexus, Buenos Aires, p. 157-171.

2. Faunt K et al. 2018. Anestesia y analgesia, Ed. Intermédica, Buenos Aires, p. 254-259.
3. Moy BT, Birk JW. 2015. Una actualización de los stents hepatobiliares. J Clin Transl Hepatol 3: 67-77.

4. Pinedo JA et al. 2008. Stents traqueo-bronquiales. Una revisión. Medigraphic Artemisa en línea 67: 117-141.

5. Rodríguez GJ, Martínez MJ, Graus MJ. 2012. Cirugía en la clínica de pequeños animales, Ed. Servet, Zaragoza, p. $238-255$.

6. Slatter D. 2006. Tratado de cirugía en pequeños animales, 3ra. ed., Ed Intermédica, Buenos Aires, p. 1005-1031. 